\title{
OPTIMIZACIÓN DE LAS CONDICIONES DE PROCESO PARA LA ELABORACIÓN DE LA ESPONJA LÍQUIDA DE PAN DE MOLDE A TRAVÉS DE UN DISEÑO FACTORIAL DE EXPERIMENTOS
}

\author{
OPTIMIZING CONDITIONS FOR THE DEVELOPMENT \\ PROCESS OF THE SPONGE LIQUID BREAD MOLD \\ THROUGH AFACTORIAL DESIGN
}

\author{
Claudia Marcela Quintero Gil', Helio Rueda Quijano²
}

\begin{abstract}
Resumen
El pan dentro de sus múltiples formas es uno de los alimentos más antiguos y ampliamente consumidos por la humanidad; su aparición no se conoce a ciencia cierta, pero se presume que fue en el oriente medio, lugar donde empezó a cultivarse el trigo.

Debido a su evolución en las diferentes culturas han sido muchos los desarrollos tecnológicos e industriales; sin embargo, todos los procesos que han sido diseñados para la fabricación del pan han tenido una finalidad común y simple: la conversión de la harina de trigo en un alimento esponjoso y apetitoso.
\end{abstract}

Este objetivo se ha logrado a través de una serie de fases comunes como son la mezcla de agua y harina, junto con la levadura, sal y otros ingredientes particulares en proporciones adecuadas, así como la generación de sabor y aroma específicos mediante la fermentación y el desarrollo de una estructura de gluten en combinación con la aplicación de energía mecánica durante el amasado y la obtención posterior de una estructura final fija y consistente con un color agradable mediante el horneado.

Para lograr este objetivo que se fundamenta en los procesos de fermentación, la industria de la panificación ha creado el término esponja para referirse a un prefermento cuya función principal es precisamente modificar el sabor y aroma y contribuir al desarrollo final de la masa mediante cambios en las propiedades reológicas de la mezcla.

Entre los ingredientes de la esponja se destaca el alimento para levadura, conocido por sus iniciales como APL, el cual tiene como función principal la de regular la fermentación mediante la combinación adecuada, tanto cualitativa, como cuantitativa, de los ingredientes.

1 Claudia Marcela Quintero Gil. Ingeniero de alimentos, Especialista en Ingeniería de Procesos en Alimentos y Biomateriales. E mail:claussquin@yahoo.com

2 Helio Rueda Quijano. Ingeniero Químico, Especialista en Ingeniería de Procesos en Alimentos y Biomateriales. E mail: helio.rueda@gmail.com 
El estudio de esta combinación se planteó en el presente trabajo con base en un diseño factorial de experimentos con tratamiento estadístico buscando evaluar los efectos de la formulación en combinación con la temperatura sobre una variable, respuesta denominada poder de gasificación la cual midió indirectamente el desarrollo de la fermentación en las esponjas líquidas que sirven como base para la preparación de pan de molde.

Los resultados arrojados permitieron definir una formulación del APL y una temperatura óptima en los cuales la variable de respuesta alcanzó los máximos valores deseados. Todo este estudio estuvo permanentemente apoyado en un método estadístico que facilitó llegar a conclusiones veraces y, además, aplicar los conocimientos adquiridos durante la especialización en Ingeniería de Procesos en Alimentos y Biomateriales.

Palabras clave: diseño factorial, APL, esponja líquida, actividad amilásica, actividad de levadura, pan de molde.

\section{Abstrac}

The bread in its many forms is one of the oldest and most widely food consumed by humanity, its appearance is not known for sure, but it is presumed that he was in the Middle East, where he began to cultivate wheat.

Due to its development in different cultures have been many developments in technology and industry, however all the processes that are designed to make bread have had a common purpose and simple, the conversion of wheat flour in a food spongy and appetizing.

This objective has been achieved through a series of stages are common as the mixture of water and flour with yeast, salt and other ingredients individuals in appropriate proportions; the generation of specific aroma and flavor through fermentation and the development a structure of gluten in combination with the application of mechanical energy during the kneading and the subsequent acquisition of a fixed and final structure consistent with a nice color through the baking.

To achieve this goal which is based on the fermentation of the baking industry has created the term to refer to a sponge pre whose primary function is precisely to change the flavor and aroma and contribute to the development of the mass through changes in the properties rheological properties of the mixture.

Among the ingredients of the sponge is drawn to the food for yeast known as (APL) that has the primary role of regulating the fermentation through the right mix of ingredients both qualitatively and quantitatively.

The study of this combination was raised in this paper using a factorial design of experiments with statistical treatment looking to evaluate the effects of the formulation in combination with the temperature on a response variable called gasification power which indirectly measured 
the development of the fermentation Liquid sponges that serve as a basis for the preparation of bread mold.

The results made it possible to define a design and the apl an optimum temperature at which the variable response reached the maximum values desired. This whole study was supported permanently on a statistical method that provided power to reach accurate conclusions, and apply the knowledge acquired during the specialization in Process Engineering in Food and biomaterials.

Key words: factorial design, APL, liquid sponge, business yeast bread mold.

Recibido: enero de 2008

Aprobado: marzo de 2008

\section{Introducción}

En la industria panadera y, especialmente, en los procesos a gran escala se ha podido identificar que uno de los retos principales es mantener una calidad consistente en los procesos y en los productos que le permita destacarse y alcanzar las metas en las ventas esperadas dentro de un rango de rentabilidad razonable.

Este reto no es fácil de superar y obliga al personal técnico de las compañías a permanecer en contacto permanente con las innovaciones tecnológicas en procesos e ingredientes y a optimizar los existentes.

Para la elaboración del pan existen varios métodos cuya diferencia radica principalmente en la forma de preparación de la masa, entre ellos: método directo, procesado rápido, desarrollo mecánico y esponja y masa; este último es el más aplicado en la industria porque con él se obtienen unas mejores propiedades reológicas de la masa, un proceso controlado, un buen desarrollo final de masa y un producto con mejores características de sabor y aroma.

En el proceso de masa con esponja se destaca el de esponjas líquidas en el cual se pueden identificar como etapas principales, la preparación, el mezclado, la fermentación y el enfriamiento; en todas ellas existen variables de control, tales como, la ingrediación, el tiempo y, especialmente, la temperatura de fermentación, la cual marca el rumbo del proceso.

Estos procesos se han venido definiendo a través de los años y están basados en conocimientos empíricos, en experiencias con otros procesos o en estudios científicos susceptibles de mejorar. Aunque se han obtenido buenos resultados, siempre mantendrán un porcentaje alto de aleatoriedad y en algunos momentos generarán fluctuaciones al proceso que se evidencian en inconsistencias en la calidad del producto.

El trabajo en mención plantea optimizar la etapa del proceso de fabricación del pan de molde, denominada esponja líquida, mediante un diseño factorial de experimentos $2^{\mathrm{k}}$, buscando optimizar una formulación del alimento para levadura (APL) y una condición inicial de proceso como es la variable temperatura, parámetros que en conjunto contribuyen a reducir 
las fluctuaciones del proceso, impactan en los costos de producción generando ahorros en el consumo de levadura y, en general, brindan una mejor calidad en el producto terminado.

\section{Materiales y métodos}

Para el estudio de las variables que tienen efecto en el proceso de gasificación de la levadura en esponjas líquidas y de acuerdo con la revisión bibliográfica se planteó inicialmente un diseño de experimentos tipo "screen design" y, posteriormente, un factorial $2^{\mathrm{k}}$, con puntos axiales tomando las variables significativas de la formulación y la variable de proceso. Se realizaron doce experimentos en la primera parte y dieciséis en la segunda con tres repeticiones cada uno más seis puntos axiales, aplicando la técnica analítica de determinación del poder de gasificación en levaduras ${ }^{3}$.

Equipos y material:

- Balanza analítica.

- Presurómetro de vasija con manómetro.

- Baño de agua a temperatura controlable.

- Utensilios: vidrio de reloj, espátula, varilla de agitación.

Procedimiento. se colocaron $100 \mathrm{~g}$ de harina (en base $14 \%$ de humedad) y la formulación en estudio en la vasija del presurómetro, la cual fue calentada a la temperatura de referencia (25 ó $27^{\circ} \mathrm{C}$ ); se mezcló perfectamente con una espátula; se roscó la tapa con el manómetro y se colocó el aparato en el baño de agua que estaba puesto previamente a la temperatura de referencia.

Después de 5 minutos, con el fin de dejar que todo el sistema adquiriera la temperatura, se ajustó el manómetro a cero abriendo la válvula por un instante y se empezó a contar el tiempo. Se tomó la lectura después de 3 horas y se reportó la presión final.

Descripción general del diseño de experimentos: una vez definida la condición de operación y la formulación se planteó el diseño de experimentos tipo "screen design" (Plackett \& Burman) buscando determinar las variables que tienen influencia y luego un factorial $2^{\mathrm{k}}$ para poder explorar en dirección óptima con puntos axiales y con repeticiones. Con esta secuencia de experimentos se buscó alcanzar el objetivo de evaluar el efecto de la formulación del APL en combinación con la temperatura de fermentación sobre la variable dependiente, "poder de gasificación de la levadura", la cual sirve como indicador de la fermentación en esponjas líquidas.

Los términos estadísticos son:

- Población: soluciones de prefermento (esponjas líquidas).

- Factores: azúcar(g), sal (g), fosfato monocálcico (g), cloruro de amonio (g), oxidante ácido ascórbico $(\mathrm{g})$, enzima alfa amilasa $(\mathrm{g})$, oxidante $\mathrm{ADA}(\mathrm{g})$, temperatura $\left({ }^{\circ} \mathrm{C}\right)$.

- Niveles: mínimo (-1) y máximo $(+1)$.

3 Técnica AACC 22-11 8E.1991. 
- Unidades experimentales: esponja líquida dentro del presurómetro.

- Variable respuesta: poder de gasificación (psi).

- Tamaño del experimento: 12 experimentos en diseño Plackett \& Burman, 16 experimentos en factorial $2^{4}$ más 8 experimentos en puntos axiales.

- Repeticiones: se hicieron tres repeticiones del factorial $2^{4}$.

\section{Resultados y discusión}

El modelo matemático obtenido en la secuencia de experimentos y que sirve de base para el análisis de superficies de respuestas es:

$$
\mathrm{R}=22.433-1.685 \mathrm{X}_{1}+1.765 \mathrm{X}_{2}+3.601 \mathrm{X}_{3}
$$

Donde:

$\mathrm{R}=$ Poder de gasificación de la levadura

$\mathrm{X}_{1}=$ Concentración de sal en la esponja líquida

$\mathrm{X}_{2}=$ Concentración de cloruro de amonio en la esponja líquida

$\mathrm{X}_{3}=$ Temperatura de fermentación de la esponja líquida

\section{Superficies de respuesta}

Del diseño factorial $2^{4}$ completo se obtuvo las siguientes superficies de respuesta para las variables con efectos estadísticamente significativos.

Superficie de respuesta del efecto de sal - cloruro de amonio. En la figura 1 se puede observar que los valores óptimos para maximizar el poder de gasificación de la levadura en esponjas líquidas, de acuerdo con la secuencia de experimentos, se encuentran en las superficies de contorno, próximas al máximo de cloruro de amonio y al mínimo de sal para los efectos en mención.

Figura 1. Superficies de respuesta Sal - Cloruro de Amonio
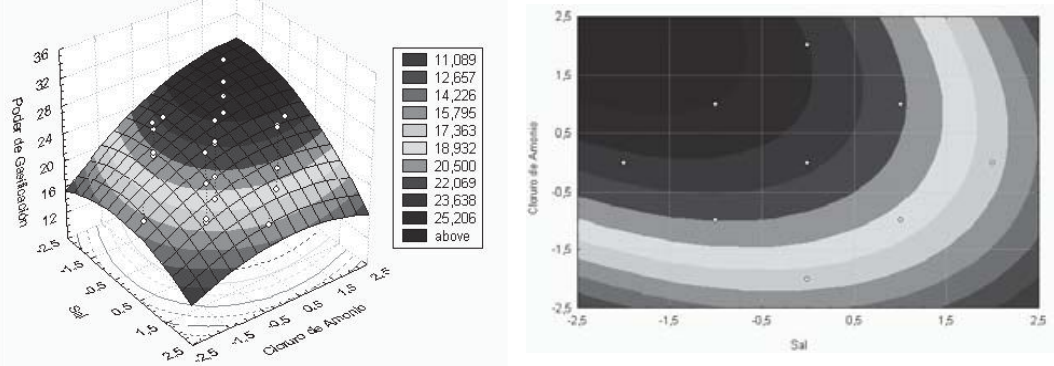
Superficie de respuesta del Efecto de Sal - Temperatura. En la figura 2 se observan los valores óptimos que permiten maximizar el poder de gasificación de la levadura en esponjas líquidas para la combinación sal - temperatura quedando la curva de contorno en la región próxima a los máximos de temperatura y los puntos medios de la concentración de sal.

Figura 2. Superficies de respuesta sal - temperatura
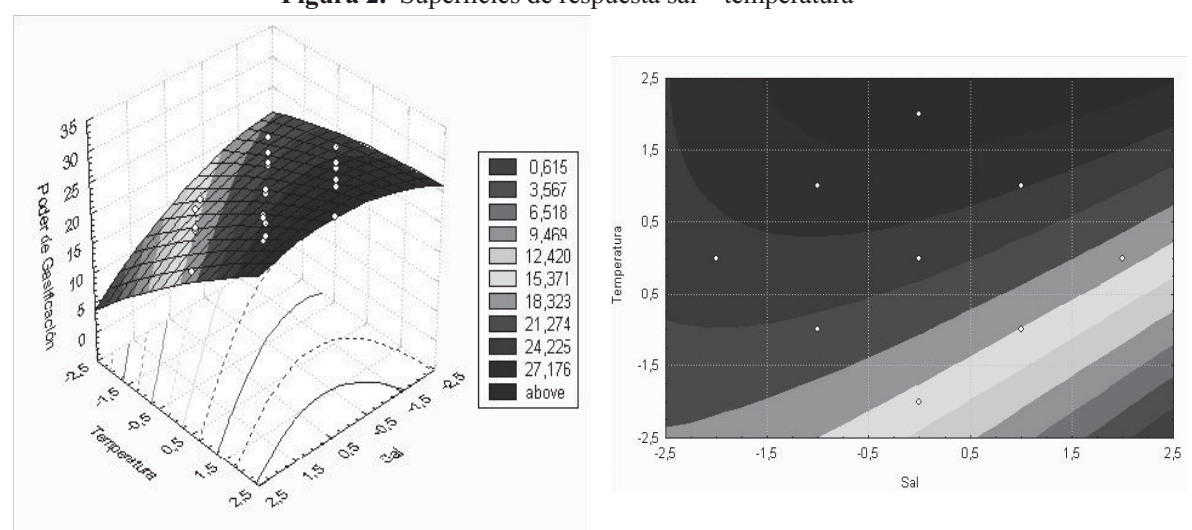

Superficie de respuesta del efecto de cloruro de amonio - Temperatura. Finalmente, en la figura 3 se observan los valores óptimos para la combinación cloruro de amonio - temperatura; estas variables permiten el máximo poder de gasificación en la curva de contorno, próxima a los máximos de las respectivas variables.

Figura 3. Superficies de respuesta cloruro de amonio - temperatura
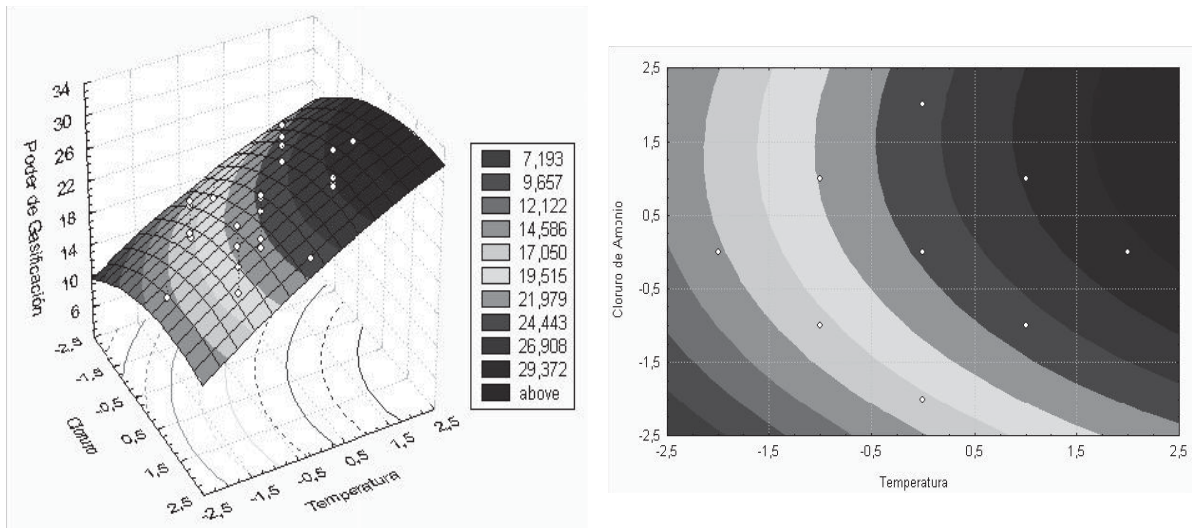


\section{Conclusiones}

- Los dos ingredientes de la formulación (sal y cloruro de amonio) y la variable de proceso (temperatura de fermentación) tienen efecto significativo sobre el poder de gasificación de la levadura en las esponjas líquidas; no sucede lo mismo con el efecto del fosfato monocálcico y la interacción entre las variables estudiadas que son estadísticamente no significativos.

- El efecto observado de la temperatura sobre el poder de gasificación de la levadura es el más representativo de todos y en magnitud pesa aproximadamente el doble que los efectos de las otras variables, lo que corrobora la información teórica del fuerte efecto que tiene sobre la fermentación y especialmente sobre la actividad de la levadura en los procesos de esponjas líquidas.

- En el diseño de experimentos se reafirma el referente teórico sobre el bajo efecto del pH en el poder de gasificación de la levadura en esponjas líquidas, ya que el ingrediente de la formulación que tuvo un efecto no significativo sobre la gasificación de la levadura fue el fosfato monocálcico cuya funcionalidad es el acondicionamiento de la acidez del agua.

- La formulación óptima de la esponja líquida para el pan de molde se obtuvo en el punto de mayor poder de gasificación (tratamiento 13 tabla 10), cuando contiene los siguientes ingredientes: $0.1 \%$ de sal, $0.1 \%$ de fosfato monocálcico, $0.2 \%$ de cloruro de amonio y se mantiene a una temperatura de fermentación en $27^{\circ} \mathrm{C}$.

- El diseño de experimentos tiene validez estadística porque cumple los supuestos del modelo como son la normalidad de los residuos y la homogeneidad de varianzas.

\section{Referencias Bibliográficas}

AMERICAN ASSOCIATION OF CEREAL CHEMISTRY (AACC). Técnica AACC 22-11 8E.1991.

ANDREWS, Graham. Technical Bulletin AIB Research Department, High Speed Dough Mixing and Mixers. Arkansas City, Kansas. December 1989.

CAUVIN, STANLEY P.; YOUNG, Linda S. Fabricación de Pan. Editorial Acribia S. A. Zaragoza. 2002.

COLLINS, T. H. Mixing, moulding and processing of bread doughs in the UK, in Breeding to Baking, Proceedings of an International Conference at FMBRA, Chorleywood, CCFRA, Chipping Campden. 1-16 June 1994, pp. 77-83.

DEPARTAMENTO DE INVESTIGACIÓN Y DESARROLLO. Curso de Panificación. Saf Mex. Toluca, Estado de México. Marzo 2003. 
DUBOIS, Donald K. Technical Bulletin AIB Research Department, Technical Assistance Industry Problems And Replies. Arkansas City, Kansas, January 1981.

KULP, Karel. Technical Bulletin AIB Research Department, Influence of Liquid Ferments on Quality Characteristics of White Pan Bread. Arkansas City, Kansas. September 1986.

MONTGOMERY, D. Diseño y Análisis de Experimentos. Limusa Wiley, 2da Edición, 2002.

RODRÍGUEZ, Maria Isabel; IEMMA, Antonio Francisco. Planejamento de Experimentos e Otimizacao de Processos. Casa do Pao Editora. 2005.

PRAT BARTES, Albert; TORT MARTORELL, Xavier. Métodos Estadísticos Control y Mejora de la Calidad. Alafaomega Edicions. 2000.

PYLER, E. J. Baking Science and Technology. Third Edition. Sosland Publishing Company, Kansas City, Missouri, 1988.

SANDERSON, Gary W; REED, Gerald; BRUINSMA, Bernard; COOPER, Elmer J. Technical Bulletin AIB Research Department, Yeast Fermentation in Bread Making. Arkansas City, Kansas. December 1983.

SCHILLER, G. W. Dixie; MILLS, Portland. Technical Bulletin AIB Research Department, Bakery Flour Specifications. Arkansas City, Kansas. February 1981.

SHARON, L. Lohr. Muestreo Diseño y Análisis. International Thomson Editores, 2000.

SPSS, Guía para el Análisis de datos. Hispano portuguesa SPSS. Luchana 23. 5ta Planta, Madrid.

VISAUTA VINACUA, B. Análisis estadístico con SPSS para Windows. Mc Graw Hill. 1998.

WALPOLE; MYERS; MYERS. Probabilidad y Estadística para Ingenieros. Pearson Education, Sexta Edición 2001.

WULF, T. Doerry; ROSS, Annabel; BAKER, Ardith. Technical Bulletin AIB Research Department, Liquid Preferments a Study of Factors,Affecting Fermentation Parameters And Bread Quality. Arkansas City, Kansas. June 1985. 